\title{
Female Genital Tuberculosis Can Cause Infertility in Women or Not?
}

\section{Shaterian $\mathbf{N}^{1}$ and Shaterian $\mathbf{N}^{2 *}$}

${ }^{1}$ Student Research Committee, J ahrom University of

Medical Sciences, J ahrom, Iran

${ }^{2}$ School of Nursing \& Midwifery, Shahid Beheshti

University of Medical Sciences, Tehran, Iran

*Correspondling author: Negin Shaterian, School of Nursing \& Midwifery, Shahid Beheshti University of Medical Sciences, Tehran, Iran

Received: August 09, 2021; Accepted: September 07, 2021; Published: September 14, 2021

\section{Letter to the Editor}

One of the important issues is infertility that is increasing today and the causes are various. Bacterium Tuberculosis is viable in pulmonary and extrapulmonary [1]. Female genital tuberculosis is an extrapulmonary type that makes up $9 \%$ of the cases [1]. Tuberculosis infection of the female genitalia leads to secondary infertility, dyspareunia, amenorrhea, irregular menstruation, and chronic pelvic inflammatory disease [2]. In $90 \%$ of cases, female genital tuberculosis affects young women aged 18 to 38 years old [3]. The global prevalence of female genital tuberculosis in infertile women is about $5 \%$ to $10 \%$ [2]. Hence, it should be considered as a differential diagnosis of the infertility causes in areas with tuberculosis infection prevalence. The gold standard method for diagnosis of this bacterium is culture, but the minimum number of bacteria should be 10-100 bacilli per milligram in this method [4]. Moreover, its long incubation period in this method leads to delays in diagnosis and treatment [4]. Also, laparoscopy can be used for diagnosis. Both laparoscopic and molecular tests are complementary tests and together they can effectively confirm the diagnosis of female genital tuberculosis [4].
For the treatment of female genital tuberculosis, multidrug therapy with rifampicin, isoniazid, pyrazinamide, and ethambutol is used, while in more severe cases, surgery is recommended [5]. However, even after using multidrug therapy, infertile women with genital tuberculosis are less likely to be fertilized, and if they do become pregnant, the consequences of pregnancy such as ectopic pregnancy or miscarriage remain high [5]. Since, untreated tuberculosis causes extrapulmonary tuberculosis, preventative measures such as BCG vaccination at birth, identification of people with tuberculosis, early diagnosis, and treatment of tuberculosis can prevent extrapulmonary tuberculosis [2]. Therefore, similar to any other disease and infection, the best way to treat and reduce this infection and then reduce the rate of infertility is to diagnose and treat it early.

\section{References}

1. Tal R, Lawal T, Granger E, Simoni M, Hui P, Buza N, et al. Genital tuberculosis screening at an academic fertility center in the United States. American Journal of Obstetrics and Gynecology. 2020; 223(5): 737. e1-. e10.

2. Shrestha A, Dhakal S. Endometrial Tuberculosis a Treatable Cause of Infertility. Kathmandu Univ Med J. 2020; 70(2): 205-206.

3. Kamal S, Singh V, Singh S. A clinical study of association of genital tuberculosis with infertility in a tertiary centre of Jharkhand, India. 2020.

4. Tiwari K, Batra S, Tanwar R, Prasad S. Comparison of Molecular Test with Hystero-Laparoscopy in Detection of Genital Tuberculosis amongst Women with Infertility. Journal of Infertility and Reproductive Biology. 2021; 9(2): 97100.

5. Wang Y, Shao R, He C, Chen L. Emerging progress on diagnosis and treatment of female genital tuberculosis. Journal of International Medical Research. 2021; 49(5): 03000605211014999. 